Pacific Journal of Mathematic 


\section{POSITIVE SOLUTIONS OF ELLIPTIC EQUATIONS}

\section{William F. Moss and John Piepenbrink}

1. Introduction. Let $\Omega$ be a domain (open, connected, possibly unbounded) in $\mathbf{R}^{n}$ and, as usual, let $x=\left(x_{1}, \cdots, x_{n}\right)$ denote a point in $\mathbf{R}^{n}$ with norm $|x|=\left(x_{1}^{2}+\cdots+x_{n}^{2}\right)^{1 / 2}$. Using the summation convention; let $L$ denote the partial differential operator defined by

$$
L u=-a_{i j}(x) u_{x_{x} x_{j}}+b_{\imath}(x) u_{x_{i}}+c(x) u .
$$

The coefficients of $L$ are assumed to be real functions defined on $\Omega$ and $a_{i j}=a_{j i}$.

If the $a_{i j}$ are continuously differentiable in $\Omega, L$ may be written in the form

$$
L u=-\left(a_{i j}(x) u_{x_{i}}\right) x_{i}+\tilde{b}_{i}(x) u_{x_{i}}+c(x) u
$$

where $\tilde{b}_{i}=b_{i}+\sum_{j=1}^{n} \partial a_{i j} / \partial x_{i}$. In this form $L$ is symmetric (formally self-adjoint) if $\tilde{b}_{i}(x) \equiv 0, i=1, \cdots, n$.

In a recent paper [1] Allegretto established the following result. Here $[r]$ denotes the integer part of the number $r$.

\section{THEOREM 1.1. Suppose that}

(A) $L$ is symmetric and the $a_{i j}$ are in class $C^{m+1}(\bar{\Omega})$ while $c$ is in $C^{m}(\bar{\Omega})$, where

$$
m=3[[3+n / 2] / 2]
$$

(B) L is elliptic in $\bar{\Omega}$, that is, $\left(a_{i j}(x)\right)$ is positive definite for each $x \in \bar{\Omega}$, and

(C) there is a number $R>0$ such that $\Omega \cap\{|x|>R\}$ is connected,

(D) for every bounded domain $D$ with $\bar{D} \subseteq \Omega \cap\{|x|>R\}$

$$
\begin{aligned}
& \inf \quad(L \phi, \phi)>0, \\
& \phi \in C_{0}^{\infty}(D) \quad(\phi, \phi) \\
& \phi \neq 0
\end{aligned}
$$

where $(\phi, \psi)=\int_{\mathbf{R}^{n}} \phi \psi d x$.

Then there exists a positive solution of $L u=0$ in $\Omega \cap\{|x|>R\}$.

If $L$ is viewed as an operator on $L_{2}(D)$ with domain $C_{0}^{\infty}(D)$, then hypothesis $(D)$ states that the smallest generalized eigenvalue of $L$ is 
greater than zero, or equivalently, that the smallest eigenvalue of the Friedrichs extension of $L$ is greater than zero. If the left hand side of (1.3) equals zero, $D$ is called a nodal domain for $L$ by I. Glazman, W. Allegretto and others.

Theorem 1.1 is interesting for two reasons. First, as Allegretto points out, it represents an extension of a property that is clearly valid for the corresponding ordinary differential operator, and at the same time clarifies the oscillation theory of symmetric, second order, elliptic operators. Second, it answers a question posed by one of the authors in [11]. There it was shown that a result such as Theorem 1.1 would imply that the finiteness of the number of negative eigenvalues of a self-adjoint realization of $L$ in $L^{2}(\Omega)$, here $\Omega=\mathbf{R}^{n}$, is invariant under perturbation of the coefficients by smooth functions with compact support. Diagrammatically we have the implications finite negative spectrum $\Rightarrow$ hypothesis $(D)$ holds for $R$ sufficiently large, and $L u=0$ has a positive solution in $\{|x|>R\} \Rightarrow$ finite negative spectrum.

The first is found in Glazman's book [5], while the second is proved in [11]. Theorem 1.1 supplies the link that makes for a closed chain of implications. It should be mentioned that the second implication was employed in [12] to unify and extend some older criteria on the potential $c(x)$ that ensured finite negative spectrum for $L=-\Delta+c(x)$.

The following result extends Theorem 1.1 to the general nonsymmetric case and at the same time weakens the smoothness required on the coefficients of $L$.

THEOREM 1.2. Let $L$ be defined by (1.1) and let the coefficients of $L$ be defined in a domain $G \subseteq \mathbf{R}^{n}$. Assume that

(a) $L$ is elliptic in $G$,

(b) the coefficients of $L$ are locally Hölder continuous in $G$,

(c) for every bounded domain $D$ with $\bar{D} \subseteq G$ the only solution in class $C^{2}(D) \cap C^{0}(\bar{D})$ to $L u=0$ in $D, u=0$ on $\dot{D}$ (the boundary of $D$ ) is $u \equiv 0$.

Then there is a positive solution $v$ of the equation $L v=0$ defined on $G$.

Allegretto's proof of Theorem 1.1 leaned heavily on the theory of symmetric quadratic forms in Hilbert space. As such it required that $L$ be symmetric. Our proof of Theorem 1.2 seems simpler and more direct. The method is a fairly straight forward application of Serrin's version of the Harnack inequality for positive solutions of second order, elliptic equations (see [13]). We also note that the smoothness required in Theorem 1.2 is mild and consonant with modern existence theories for elliptic boundary value problems. Thus it represents a distinct sharpening of Theorem 1.1 even in the symmetric case. 
In Theorem $1.1 \infty$ is the only possible point at which $L$ may degenerate or the coefficients of $L$ become unbounded. In Theorem 1.2 any boundary point may have this property. There is no significant difference here. Assuming $L$ is symmetric and that (a) and (b) hold, (c) is equivalent to $(D)$ with $\Omega \cap\{|x|>R\}$ replaced by $G$. This will be discussed further in $\$ 3$. Hypothesis $(D)$ is more easily used in the Hilbert space context, while (c) is best adapted for our proof of Theorem 1.2.

2. Preliminary lemmas. The proof of Theorem 1.2 uses the maximum principle, the Harnack inequality, and the Schauder existence theory. The first two of these are stated in the following lemmas. For the Schauder existence theory the reader is referred to Miranda [10]. In Lemmas 2.1 and $2.2 L$ is given by (1.1) and the coefficients of $L$ are defined on a domain $D$ in $\mathbf{R}^{n}$.

Lemma 2.1. Assume that

(i) there is a positive constant $\mu$ such that $a_{i j}(x) \xi_{i} \xi_{j} \geqq \mu, x \in D$, $|\xi|=1$,

(ii) $a_{i i}$ and $b_{i}, i=1, \cdots, n$, are bounded in $D$ and $c \geqq 0$ in $D$. If $u \in C^{2}(D), L u \geqq 0$ in $D$, and there exists $x_{0} \in D$ such that $\inf _{G} u=u\left(x_{0}\right) \leqq$ 0 , then $u \equiv$ constant.

Corollary. If $u \in C^{2}(G), L u \geqq 0$ in $G, u \geqq 0$ in $G$ and $u \neq 0$, then $u>0$ in $G$.

With $u\left(x_{0}\right) \leqq 0$ replaced by $u\left(x_{0}\right)<0$, this is a well known result due to E. Hopf [6]. In case $u\left(x_{0}\right)=0$, the boundary point principle of $\mathrm{G}$. Giraud [4] can be applied (see also [10], pp. 6-7 and [2], pp. 150-152).

The following extension of the classical Harnack inequality for positive harmonic functions is due to Serrin [13].

LemMA 2.2. Suppose there exist positive constants $\mu$ and $M$ and a continuous, nondecreasing function $\phi$ with

$$
\int_{0^{+}}^{\infty} \frac{\phi(s)}{s} d s<+\infty
$$

such that for $x, y \in D$

(i) $\mu \leqq a_{i j}(x) \xi_{i} \xi_{j} \leqq \mu^{-1},|\xi|=1$,

(ii) $\left(\sum_{i=1}^{n} b_{i}^{2}(x)\right)^{1 / 2} \leqq M$,

(iii) $0 \leqq c(x) \leqq M$,

(iv) $\left|a_{i j}(x)-a_{i j}(y)\right| \leqq \phi(|x-y|)$.

Then for any bounded domain $D_{0} \bar{D}_{0} \subset D$, there is a constant $K=K\left(\mu, M, \phi, D_{0}, D\right)$ so that for each positive solution $u$ of $L u=0$ in $D$, 


$$
K^{-1} u(y) \leqq u(x) \leqq K u(y), \quad x, y \in D_{0} .
$$

It is interesting to note that Serrin's proof uses only the maximum principle and a suitable parametrix, and that Serrin showed in case $n=2$ that hypothesis (iv) is unnecessary. We shall not be concerned with this refinement. What will be important here is the following.

COROllary. Replace (iii) by

(iii)' There is a $v$ in $C^{2}(\bar{D})$ so that $v>0$ and $L v \geqq 0$ on $\bar{D}$.

Then (2.1) still holds, where $K$ may depend on $v$ as well as the other parameters.

For the proof set $\hat{u}=u / v$ and apply Serrin's theorem with $L$ and $u$ replaced by $\hat{L}$ and $\hat{u}$. $\hat{L}$ is defined by

$$
\hat{L} \hat{u}=-a_{i j} \hat{u}_{x_{i} x_{j}}+\left(b_{i}-2 a_{i j} v_{x_{j}} / v\right) \hat{u}_{x_{i}}+(L v / v) \hat{u} .
$$

Since $L u=0$, it follows that $\hat{L} \hat{u}=0$ since $\hat{L} \hat{u}=L u / v$. Thus there is a constant $\hat{K}$ such that

$$
\hat{K}^{-1} \hat{u}(y) \leqq \hat{u}(x) \leqq \hat{K} \hat{u}(y) .
$$

From the definition of $\hat{u}$ we can easily see that (2.1) holds if we set

$$
K=\frac{\max _{D_{0}} v}{\min _{D_{0}} v} \hat{K} .
$$

We now apply the Schauder existence theory to prove

Lemma 2.3. Suppose the hypotheses of Theorem 2.1 hold. Then for every bounded domain $F$ with $\vec{F} \subseteq G$ and $\dot{F}$ sufficiently smooth, there is a solution $v \in C^{2}(\bar{F})$ to $L u=0$ in $\bar{F}$ which is positive on $\bar{F}$.

Proof. According to hypothesis (b) the coefficients of $L$ are Hölder continuous in $\bar{F}$. Let $\alpha=\alpha(F)$ denote the minimum of the Hölder exponents in $\bar{F}$ of the coefficients of $L$. We assume that $\dot{F}$ is sufficiently smooth so that $\dot{F} \in C^{2+\alpha}$. Then the Schauder existence theory shows that there exists a function

$$
\begin{aligned}
v & \in C^{2+\alpha}(\bar{F}) \\
L v & =0 \text { in } F \\
v & =1 \text { on } \dot{F} .
\end{aligned}
$$

More specifically, a result of Boboc and Mustata [3] shows that there is a positive constant $\gamma=\gamma(F)$ such that if $u \in C^{2}(F) \cap C^{0}(\bar{F})$, then 


$$
\|u\|_{0}^{F} \leqq \gamma\left[\|L u\|_{0}^{F}+\|u\|_{0}^{\dot{F}}\right]
$$

This estimate can be used in conjunction with the classical Schauder estimate up to the boundary to prove the existence of solutions to Dirichlet problems such as (2.2) (see, for example, [10], p. 166, 36, II).

Now we claim that $v$ is positive. First, $v \geqq 0$ on $F$ for otherwise let $O$ denote some connected component of $\{x \in F: v(x)<0\}$. Then $v<0$ in $O, L v=0$ in $O, v=0$ on $\dot{O}$, a contradiction to (c). But since $v \geqq 0$ on $F$,

$$
-a_{i j} v_{x_{1}, x_{j}}+b_{i} v_{x_{i}}+c^{+} v=c^{-} v \geqq 0 \text { on } F,
$$

where $c^{+}(x)=\max \{c(x), 0\}$ and $c^{-}(x) \equiv c^{+}(x)-c(x)$, so that the corollary to Lemma 2.1 implies that $v>0$ on $F$.

3. Proof of Theorem 2.2. There exists a sequence of bounded domains in $\mathbf{R}^{n}$ with analytic boundaries such that $\bar{G}_{k} \subseteq G_{k+1} \subseteq \bar{G}_{k+1} \subseteq G$, $k=1,2, \cdots$, and $G=\bigcap_{k=1}^{\infty} G_{k}$ (see, for example [7], pp. 317-319). Let $\alpha_{k}$ denote the minimum of the Hölder exponents in $\bar{G}_{k}$ of the coefficients of $L$. Furthermore, let $u_{k}$ denote the positive solution to

$$
\begin{aligned}
u_{k} & \in C^{2+\alpha_{k}}\left(\bar{G}_{k}\right) \\
L u_{k} & =0 \text { in } G_{k} \\
u_{k} & =1 \text { on } \dot{G}_{k}
\end{aligned}
$$

which exists according to Lemma 2.3.

Now choose $x_{0} \in G_{1}$ and let $v_{k}=u_{k} / u_{k}\left(x_{0}\right)$. Next, apply the corollary to Lemma 2.2 with $D_{0}=G_{k}, D=G_{k+1}, v=v_{k+1}$ and $u=v_{l}, l \geqq$ $k+1$. Then there are positive constants $m_{k}$ and $M_{k}$ so that

$$
m_{k} \leqq v_{l}(x) \leqq M_{k}, \quad x \in \bar{G}_{k}, \quad l \geqq k+1 .
$$

According to the Schauder estimates plus Ascoli's theorem, $\left\{v_{l}\right\}_{l \geqq k+1}$ has a subsequence which converges in $C^{2+\alpha_{k}}\left(\bar{G}_{k}\right)$. By a diagonalization process a subsequence of $\left\{v_{k}\right\}_{k \geqq 1}$ is obtained which converges uniformly on every compact subset of $G$ to a function $v \in C^{2+\alpha_{k}}\left(\bar{G}_{k}\right), k=1,2, \cdots$, with $L u=0$ in $G$. Also $m_{k} \leqq v \leqq M_{k}$ on $\bar{G}_{k}$ and $v\left(x_{0}\right)=1$. Thus the desired positive solution exists.

In $\S 1$ we stated that assuming $L$ is symmetric and (a) and (b) hold, (c) is equivalent to (D) with $\Omega \cap\{|x|>R\}$ replaced by $G$. To see that (c) implies (D) let $D$ be a domain as in (D) and let $F$ be a bounded, smooth domain with $\bar{D} \subseteq F \subseteq \bar{F} \subseteq G$. By Lemma 2.3 there is a function $v \in$ $C^{2}(\bar{F}), v>0$ on $\bar{F}$ and $L v=0$ in $F$. Let $\phi \in C_{0}^{\infty}(D)$ and set $\tilde{\phi}=$ 
$\phi / v$. Then $\tilde{\phi} \in C_{0}^{2}(D)$. Applying Green's theorem several times we obtain

$$
\frac{(L \phi, \phi)}{(\phi, \phi)}=\frac{\int_{D} v^{2} a_{i j} \tilde{\phi}_{x_{i}} \tilde{\phi}_{x_{1}} d x}{\int_{D} \tilde{\phi}^{2} v^{2} d x} .
$$

Hence

$$
\frac{(L \phi, \phi)}{(\phi, \phi)} \geqq M \frac{\int_{D} \sum_{i=1}^{n} \tilde{\phi}_{x_{1}}^{2} d x}{\int_{D} \tilde{\phi}^{2} d x} \geqq M C,
$$

where

$$
M=\frac{\min _{D} v^{2}}{\max _{D} v^{2}} \min _{\bar{D} x\{|\xi|=1\}} a_{i j}(x) \xi_{i} \xi_{j},
$$

and $C$ is the constant in Friedrichs' inequality which depends only on $D$ and not on $\tilde{\phi}$ (see, for example, [9], p. 290).

To see that (D) $\Rightarrow(\mathrm{c})$, again let $D$ and $F$ be domains as above. Consider $L$ as an operator on $L_{2}(F)$ with domain $C_{0}^{\infty}(F)$, and choose a nonnegative function $f \not \equiv 0$ in $C_{0}^{\infty}(F)$. Then the equation $L u=f$ has a unique generalized solution $v$ in $H_{0}^{1}(F)$. Following Allegretto and others, it can be shown that $f \geqq 0$ implies $v \geqq 0$ a.e. in $F$. Since for smooth $F$,

$$
\left.\left\{u \in C^{2+\alpha}(\bar{F}): u=0 \text { on } \dot{F}\right\} \subseteq H_{0}^{1}(F) \text { ( } \alpha \text { may depend on } F\right),
$$

it follows that the Dirichlet problem

$$
\begin{aligned}
u & \in C^{2+\alpha}(\bar{F}) \\
L u & =f \text { in } F \\
u & =0 \text { on } \dot{F}
\end{aligned}
$$

has at most one solution. But this implies existence according to the general Schauder theory (see [10], p. 166, 36, II), so that $v$ can be identified with the solution to (3.1). Since $v$ satisfies (2.3), the corollary to Lemma 2.1 implies that $v>0$ on $F$. But then the generalized maximum principle (see [10], p. 163, 35, IX) implies that the problem 
$L u=0$ in $D, u=0$ on $\dot{D}$ has at most one solution in class $C^{2}(D) \cap$ $C^{0}(\bar{D})$.

4. Conclusion. An example in Allegretto's paper ([1], p. 324) has led us to formulate the following corollary of Theorem 1.2.

Corollary. Consider the operator $L$ defined by

$$
-L=\Delta+p
$$

where $p$ is nonnegative and continuous on $\mathbf{R}^{n}$. If $p \not \equiv 0$, then $L$ has $a$ nodal domain in $\mathbf{R}^{2}$.

Proof. We can assume without loss of generality that $p$ is locally Hölder continuous. Otherwise, choose such a $\tilde{p}$ with $0 \leqq \tilde{p} \leqq p$ and $\tilde{p} \not \equiv 0$. Then by comparison of quadratic forms, the existence of a nodal domain for $-\Delta-\tilde{p}$ implies the same for $L$.

Now by Theorem 1.2 if no nodal domain existed, we could find a positive $v \in C^{2}\left(\mathbf{R}^{2}\right)$ with $L v=0$. But then $\Delta v=-p v \leqq 0$ and $v>0$ would imply by Liouville's theorem that $v \equiv$ constant. But then $p \equiv 0$ would necessarily follow.

There is a direct computational proof of this corollary. By translation of coordinates, we may assume that $p(x) \geqq p_{0}$ for $|x| \leqq r_{0}$, where $p_{0}$ and $r_{0}$ are positive constants. It suffices to produce a nodal domain for the equation

$$
\Delta u+\tilde{p}(r) u=0, \quad r=|x|,
$$

where $\tilde{p}(r)$ is continuous and $0 \leqq \tilde{p}(|x|) \leqq p(x)$ for all $x$ and $\tilde{p}(r)=p_{0}$ for $0 \leqq r \leqq r_{0}$. If $\phi(r)$ is the solution to the initial value problem

$$
\begin{gathered}
\phi^{\prime \prime}(r)+\frac{1}{r} \phi^{\prime}(r)+\tilde{p}(r) \phi(r)=0, \quad r>0 \\
\phi(0)=1, \quad \phi^{\prime}(0)=0,
\end{gathered}
$$

then $v(x)=\phi(|x|)$ is a regular solution of (4.1). We prove that there is an $R>0$ such that $\phi(R)=0$. Thus $v(x)=\phi(|x|)$ satisfies (4.1) and $\{|x|<R\}$ is a nodal domain for (4.1). There will then be a nodal domain for $L$ contained in $\{|x|<R\}$.

Since $r=0$ is a regular singular point for (4.2), we can compute the series

$$
\phi(r)=1-\frac{1}{4} p_{0} r^{2}+\cdots
$$


which is valid at least for $0 \leqq r<r_{0}$. Clearly, we can find an $r_{1}, 0<r_{1}<r_{0}$ so that $\phi^{\prime}\left(r_{1}\right)<0$. Now if $\phi(r)>0$ for all $r>0, \phi$ would satisfy the inequality

$$
\left(r \phi^{\prime}(r)\right)^{\prime} \leqq 0, \quad r \geqq 0
$$

Integrating, we have

$$
r \phi^{\prime}(r) \leqq r_{1}^{\prime} \phi^{\prime}\left(r_{1}\right) \equiv-\alpha<0, \quad r \geqq r_{1} .
$$

Thus

$$
0<\phi(r) \leqq \phi\left(r_{1}\right)+\alpha \log \frac{r_{1}}{r}, \quad r \geqq r_{1}
$$

\section{A contradiction arises for sufficiently large $r$.}

\section{REFERENCES}

1. W. Allegretto, On the equivalence of two types of oscillation for elliptic operators, Pacific J. Math., 55 (1974), 319-328.

2. L. Bers, F. John and M. Schechter, Partial Differential Equations, Amer. Math. Soc., Providence R.I., 1964.

3. Boboc and Mustata, Sur un probleme concernant les domaines d'unicité pour le probleme de Dirichlet associé á un operateur elliptique, Rend. Acc. Naz. Lincei, 42 (1967).

4. G. Giraud, Generalisation des problemes sur les operations du type elliptique, Bull. Sci. Math., 56 (1932), 316-352.

5. I. M. Glazman, Direct Methods of Qualitative Spectral Analysis of Singular Differential Operators, Daniel Davey and Co., Inc., New York, 1965.

6. E. Hopf, Elementare Betrachtungen über die Lösungen partieller Differentialgleichungen Zweiter Ordnung vom elliptichen Typus, Sitzungsbericht preuss. Akad. Wiss., 19 (1927), 147-152.

7. O. D. Kellogg, Foundations of Potential Theory, Dover, New York, 1953.

8. S. G. Mikhlin, The Problem of The Minimum of A Quadratic Functional, Holden-Day, San Francisco, 1965.

9. _- Mathematical Physics, An Advanced Course, North-Holland, London, 1970.

10. C. Miranda, Partial Differential Equations of Elliptic Type, 2nd. ed., Springer, New York, 1970.

11. John Piepenbrink, Nonoscillatory elliptic equations, J. Differential Equations, 15 (1974), 541-550.

12. - Finiteness of the lower spectrum of Schrödinger operators, Math. Z., 140 (1974), 29-40.

13. James Serrin, On the Harnack inequality for linear elliptic equations, J. Analyse Math., 4 (1955/56), 297-308.

Received February 2, 1977 and in revised form June 23, 1977. Supported in part by the Georgia Tech. Foundation. 


\section{PACIFIC JOURNAL OF MATHEMATICS EDITORS}

ICHARD ARENS (Managing Editor)

niversity of California

os Angeles, CA 90024

\section{. A. BEAUmont}

niversity of Washington

sattle, WA 98105

. C. MOORE

niversity of California

erkeley, CA 94720
J. DUGUNDJI

Department of Mathematics

University of Southern California

Los Angeles, CA 90007

R. FINN AND J. MiLgRAM

Stanford University

Stanford, CA 94305

\section{ASSOCIATE EDITORS}
F. BECKENBACH
B. H. NEUMANN
F. WOLF
K. YosHidA

\section{SUPPORTING INSTITUTIONS}

NIVERSITY OF BRITISH COLUMBIA

UNIVERSITY OF SOUTHERN CALIFORNIA

ALIFORNIA INSTITUTE OF TECHNOLOGY STANFORD UNIVERSITY

NIVERSITY OF CALIFORNIA

ONTANA STATE UNIVERSITY

UNIVERSITY OF HAWAII

NIVERSITY OF NEVADA

UNIVERSITY OF TOKYO

EW MEXICO STATE UNIVERSITY

UNIVERSITY OF UTAH

REGON STATE UNIVERSITY

NIVERSITY OF OREGON

WASHINGTON STATE UNIVERSITY

UNIVERSITY OF WASHINGTON

SAKA UNIVERSITY

AMERICAN MATHEMATICAL SOCIETY

The Supporting Institutions listed above contribute to the cost of publication of this Journal, but they a t owners or publishers and have no responsibility for its contents or policies.

Mathematical papers intended for publication in the Pacific Journal of Mathematics should be in typ Irm or offset-reproduced (not dittoed), double spaced with large margins. Underline Greek letters in re ierman in green, and script in blue. The first paragraph or two must be capable of being used separately as 'nopsis of the entire paper. Items of the bibliography should not be cited there unless absolutely necessary, hich case they must be identified by author and Journal, rather than by item number. Manuscripts, uplicate, may be sent to any one of the four editors. Please classify according to the scheme of Math. Revieu Idex to Vol. 39. All other communications should be addressed to the managing editor, or Elaine Bart Iniversity of California, Los Angeles, California, 90024.

100 reprints are provided free for each article, only if page charges have been substantially pai dditional copies may be obtained at cost in multiples of 50 .

The Pacific Journal of Mathematics is issued monthly as of January 1966. Regular subscription rate: \$72. year (6 Vols., 12 issues). Special rate: $\$ 36.00$ a year to individual members of supporting institutions.

Subscriptions, orders for numbers issued in the last three calendar years, and changes of address should ent to Pacific Journal of Mathematics, 103 Highland Boulevard, Berkeley, California, 94708.

UBLISHED BY PACIFIC JOURNAL OF MATHEMATICS, A NON-PROFIT CORPORATION

Printed at Jerusalem Academic Press, POB 2390, Jerusalem, Israel.

Copyright (C) 1978 Pacific Journal of Mathematics

All Rights Reserved 


\section{Pacific Journal of Mathematics}

\section{Vol. 75, No. $1 \quad$ September, 1978}

Mieczyslaw Altman, General solvability theorems

Denise Amar and Eric Amar, Sur les suites d'interpolation en plusieurs variables ..........................................

Herbert Stanley Bear, Jr. and Gerald Norman Hile, Algebras which satisfy a second order linear partial differential equation ..................

Marilyn Breen, Sets in $R^{d}$ having $(d-2)$-dimensional kernels ............

Gavin Brown and William Moran, Analytic discs in the maximal ideal space

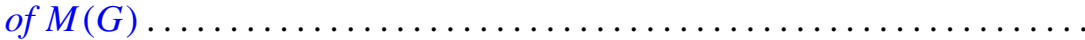

Ronald P. Brown, Quadratic forms with prescribed Stiefel-Whitney

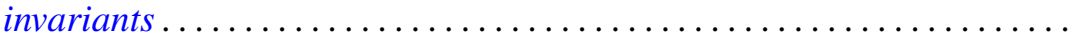

Gulbank D. Chakerian and H. Groemer, On coverings of Euclidean space by convex sets

S. Feigelstock and Z. Schlussel, Principal ideal and Noetherian groups.....

Ralph S. Freese and James Bryant Nation, Projective lattices ............

Harry Gingold, Uniqueness of linear boundary value problems for

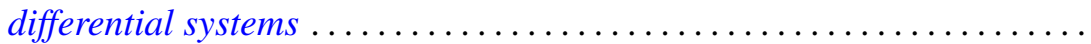

John R. Hedstrom and Evan Green Houston, Jr., Pseudo-valuation domains. . .

William Josephson, Coallocation between lattices with applications to measure extensions

M. Koskela, A characterization of non-negative matrix operators on $l^{p}$ to $l^{q}$ with $\infty>p \geq q>1$

Kurt Kreith and Charles Andrew Swanson, Conjugate points for nonlinear differential equations...........................

Shoji Kyuno, On prime gamma rings ........................ 185

Alois Andreas Lechicki, On bounded and subcontinuous multifunctions ..

Roberto Longo, A simple proof of the existence of modular automorphisms in approximately finite-dimensional von Neumann algebras ...

Kenneth Millett, Obstructions to pseudoisotopy implying isotopy for

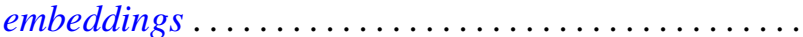

William F. Moss and John Piepenbrink, Positive solutions of elliptic equations. .

Mitsuru Nakai and Leo Sario, Duffin's function and Hadamard's

conjecture

Mohan S. Putcha, Word equations in some geometric semigroups ...

Walter Rudin, Peak-interpolation sets of class $C^{1} \ldots \ldots \ldots$

Elias Saab, On the Radon-Nikodým property in a class of locally convex

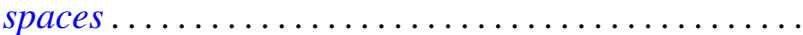

